\title{
Magnetic confinement in the solar interior
}

\author{
Toby S. Wood \\ Baskin School of Engineering, University of California Santa Cruz \\ 1156 High Street, Santa Cruz, CA 95064 \\ email: tsw25@soe.ucsc.edu
}

\begin{abstract}
The observed uniform rotation of the Sun's radiative interior can be explained by the presence of a global-scale interior magnetic field, provided that the field remains confined below the convection zone. In high latitudes, such magnetic confinement is possible by means of persistent downwelling, driven by the convection zone's turbulent stresses.
\end{abstract}

Keywords. MHD, Sun: abundances, Sun: interior, Sun: magnetic fields, Sun: rotation

\section{Introduction}

Within the solar convection zone turbulent stresses drive differential rotation (Fig. 1) and "gyroscopically pump" mean meridional circulations (MMCs) that burrow into the radiative interior (Spiegel \& Zahn 1992). These MMCs transport angular momentum below the base of the convection zone, yet the differential rotation goes over into uniform rotation within the radiative interior, via a thin shear layer called the "tachocline".

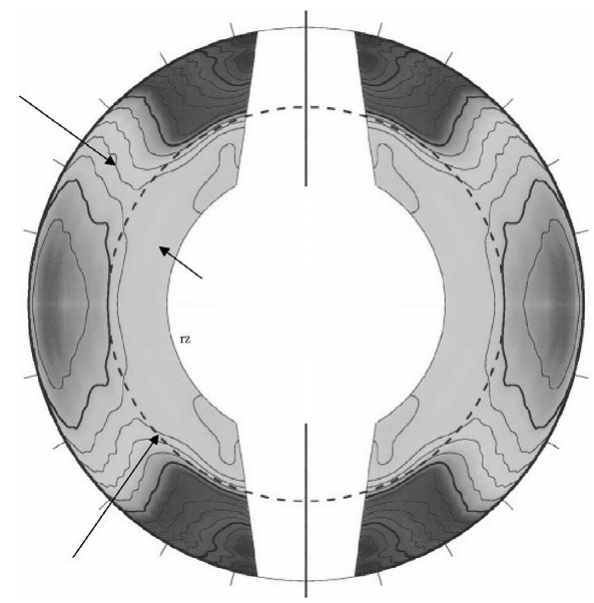

Figure 1. The Sun's differential rotation, adapted from Schou et al. (1998). The radiative interior rotates approximately uniformly with angular velocity $\Omega_{\mathrm{i}}=2.7 \times 10^{-6} \mathrm{~s}^{-1}$, or $435 \mathrm{nHz}$. Within the convection zone, the angular velocity increases with colatitude through 350,400 , $450 \mathrm{nHz}$ (heavy contours) to a maximum just under $470 \mathrm{nHz}$ at the equator.

The uniform interior rotation can be explained by the presence of a global-scale interior magnetic field $\mathbf{B}_{\mathrm{i}}$, in accordance with Ferraro's Law. To be effective, the field lines must directly couple low and high latitudes within the radiative interior; we refer to such a field as "confined". In order for $\mathbf{B}_{i}$ to remain confined over the Sun's lifetime, there needs to be some mechanism that can counteract the diffusion of $\mathbf{B}_{\mathrm{i}}$ into the convection zone (Gough \& McIntyre 1998, hereafter GM98). 
For simplicity we suppose that $\mathbf{B}_{\mathrm{i}}$ is roughly an axial dipole. In low latitudes, where the field lines are nearly horizontal, we assume that $\mathbf{B}_{i}$ is confined by downward "magnetic flux pumping" in the convective overshoot layer (e.g. Tobias et al. 2001; Kitchatinov \& Rüdiger 2008). Downward pumping of this kind is believed to act only on the horizontal components of the magnetic field, and is therefore less plausible as a confinement mechanism in high latitudes. However, the tachocline's MMCs are expected to be downwelling in high latitudes, suggesting that $\mathbf{B}_{\mathrm{i}}$ can be held in advective-diffusive balance across a thin "magnetic confinement layer" at the bottom of the tachocline (GM98).

\section{The magnetic confinement layer}

We seek to model the confinement layer in a neighborhood of the north pole (Fig. 2). We compute axisymmetric, steady-state solutions to the nonlinear, Boussinesq MHD equations using a finite-difference code.

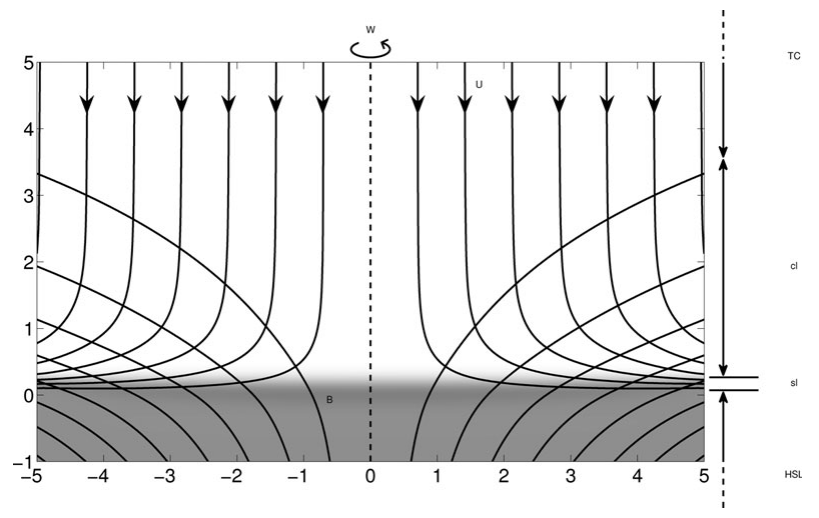

Figure 2. A vertical cross-section through the magnetic confinement layer near the north pole. The streamlines with arrows show the downwelling MMC responsible for confining the magnetic field B. Over the Sun's lifetime, helium settles out of the convection zone and establishes a stable compositional gradient within the outer part of the radiative interior. The helium settling layer (shaded) is almost impermeable, and so the downwelling MMC turns equatorward within a thin "helium sublayer". For solar magnetic and helium diffusivities the sublayer is thinner than the confinement layer by about an order of magnitude (Wood \& McIntyre 2010).

We find that downwelling of magnitude $\sim 10^{-5} \mathrm{~cm} \mathrm{~s}^{-1}$ is able to confine a broad range of interior field strengths $\left(\left|\mathbf{B}_{\mathrm{i}}\right| \lesssim 500 \mathrm{G}\right)$ across a confinement layer of thickness $\sim 1 \mathrm{Mm}$. The retrograde Coriolis torque from the equatorward flow is balanced almost exactly by a prograde Lorentz torque from the confined magnetic field, with viscosity playing almost no role. The solutions are magnetostrophic to excellent approximation.

Our results also show the importance of compositional stratification for the structure of the confinement layer. Further details, and a discussion of the implications for solar lithium depletion, will appear in a future publication (Wood \& McIntyre 2010).

\section{References}

Gough, D. O. \& McIntyre, M. E. 1998, Nature, 394, 755

Kitchatinov, L. L. \& Rüdiger, G. 2008, AN, 329, 372

Schou, J., et al. 1998, ApJ, 505, 390

Spiegel, E. A. \& Zahn, J.-P. 1992, A\&A, 265, 106

Tobias, S. M., Brummell, N. H., Clune, T. L., \& Toomre, J. 2001, ApJ, 549, 1183-1203

Wood, T. S. \& McIntyre, M. E. 2010, J. Fluid Mech., submitted 